\title{
COMMUNICATION
}

\section{Transformation maligne d'un lichen plan buccal en carcinome cuniculatum : à propos d'un cas}

\author{
Dubourg $\mathbf{S}^{1}$, Sellam E1, Schultz $\mathrm{P}^{2}$, Marcellin $\mathrm{L}^{3}$, Gros $\mathrm{C}^{1-4}$, Riehm $\mathrm{S}^{5}$, Huart $A^{6}$, Bornert $\mathrm{F}^{1}$ \\ 1 - Unité de Pathologie-Chirurgie buccale, Pôle de Médecine et de Chirurgie bucco-dentaires, Hôpital Civil, \\ Hôpitaux Universitaires de Strasbourg \\ 2 - Service d'ORL, Hôpital de Hautepierre, Hôpitaux Universitaires de Strasbourg \\ 3 - Département de Pathologie, Hôpital de Hautepierre, Hôpitaux Universitaires de Strasbourg \\ 4 - Unité de Radiologie, Pôle de Médecine et de Chirurgie bucco-dentaires, Hôpital Civil, Hôpitaux Universitaires \\ de Strasbourg \\ 5 - Service de Radiologie et d'Imagerie Médicale, Hôpital de Hautepierre, Hôpitaux Universitaires de Strasbourg \\ 6 - Service de Stomatologie - Chirurgie Maxillo-Faciale-Plastique et Reconstructrice, Hôpital Civil, Hôpitaux \\ Universitaires de Strasbourg
}

Le lichen plan est une dermatose chronique inflammatoire de la peau, des phanères et des muqueuses, qui touche fréquemment la muqueuse orale. Son étiologie demeure inconnue et la pathogénie actuellement admise est celle d'une maladie auto-immune à médiation cellulaire. Les atteintes cutanées semblent moins fréquentes que les atteintes de la muqueuse orale mais les deux formes peuvent coexister.

Chez 30 à $70 \%$ des malades ayant un lichen plan cutané on retrouve une atteinte muqueuse associée. Le lichen plan buccal isolé représente $25 \%$ des cas de lichen plan. Une prédominance féminine est fortement observée allant jusqu'à $75 \%$ des cas. L'incidence du lichen plan buccal varie, selon les auteurs, de $0,1 \%$ à $4 \%$, avec une prédilection pour les sujets d'âge moyen, entre 30 et 60 ans.

Contrairement à la forme cutanée, les lésions de la muqueuse buccale semblent persister tout au long de la vie et une rémission spontanée semble être exceptionnelle. Ces lésions peuvent évoluer et un même individu peut présenter une à plusieurs formes cliniques en même temps. Les différentes formes évoluent généralement vers un état cicatriciel, dit état post-lichénien, d'aspect variable, comportant un risque d'évolution carcinomateuse à long terme.

De nombreuses études tentent de mettre en évidence un risque de transformation maligne du lichen plan buccal montrant un taux variant de 0 à $10 \%$. De ce fait, les praticiens de la sphère orale sont en première ligne pour le diagnostic précoce des lésions malignes.

Une patiente de 70 ans s'est présentée adressée par son chirurgien-dentiste pour une lésion suspecte mandibulaire qui s'est avérée être un carcinome cuniculatum (CC) ayant nécessité une mandibulectomie interruptrice avec reconstruction par un lambeau libre. L'historique de la patiente a révélé l'existence d'un lichen plan gingival connu depuis environ 8 ans en regard de la région du CC. 
L'ensemble des aspects cliniques et radiologiques seront présentés avec une discussion autour du risque de transformation maligne du lichen plan buccal et de cette forme clinique peu fréquente du carcinome cuniculatum avec envahissement mandibulaire.

Ce cas souligne l'obligation d'information et de suivi à long terme du patient présentant un lichen plan buccal.

\section{Références}

Lombardi T, Küffer R. Concept actuel du lichen plan oral. Le diagnostic facile au début, peut devenir très difficile dans les lichens anciens. Presse Med. 2015 Nov 17

Carbone M, Arduino PG, Carrozzo M, Gandolfo S, Argiolas MR, Bertolusso G, Conrotto D, Pentenero M, Broccoletti R. Course of oral lichen planus: a retrospective study of 808 northern Italian patients. Oral Dis. 2009 Apr;15(3):235-43 Shapiro MC, Wong B, O’Brien MJ, Salama A. Mandibular Destruction Secondary to Invasion by Carcinoma Cuniculatum. J Oral Maxillofac Surg. 2015 Dec;73(12):2343-51 